\title{
CURRENT DISTRIBUTION IN SUPRACONDUCTORS
}

\author{
By F. B. Silsbee
}

ABSTRACT

This paper analyzes in detail the electrical conditions to be expected when a tube of supraconducting material is subjected to the action of the magnetic field both from a current flowing in the tube and also from a current flowing in a wire lying along the axis of the tube. The results are compared with the data experimentally obtained at Leiden by Kamerlingh Onnes and his coworkers. The agreement found supports the assumptions on which the theoretical work is based.

\section{CONTENTS}

I. Introduction .

II. Calculations of current distribution ...

III. Comparison with experiments of Onnes and Tuyn on tubular specimens_................... 301

1. Experiments of March 12, 1924_....... 301

2. Experiments of April 17, 1924

V. Appendix ........ 306

\section{INTRODUCTION}

It has been found by H. Kamerlingh Onnes ${ }^{1}$ at Leiden that as certain metals (such as mercury, lead, and tin) are cooled down from room temperature their electrical resistivity decreases uniformly with the normal coefficient of about 0.4 per cent per degree until the temperature is very low, when the rate of decrease becomes less rapid. At a certain critical temperature $\left(T_{\mathrm{c}}\right)$, however, there is an abrupt change and, within a temperature range of a few hundredths of a degree, the resistivity drops to less than $10^{-12}$ times its value at $0^{\circ} \mathrm{C}$. It was also found ${ }^{2}$ that if the temperature of the bath was held at a constant value somewhat lower than the critical temperature, while the current through the specimen was increased gradually, the normal resistance of the specimen reappeared suddenly at a certain "threshold" value of current $\left(I_{\mathrm{c}}\right)$. The lower the temperature the greater $I_{\mathrm{c}}$.

It was further found that if the specimen at a temperature below $T_{\mathrm{c}}$ was placed in a magnetic field which was gradually increased in intensity the normal resistance reappeared at a certain critical value $\left(H_{\mathrm{c}}\right)$ of the magnetic field. ${ }^{3}$

\footnotetext{
1 Proc. Kon. Akad. v. Weten., Amsterdam, 17, 1, p. 280.

2 Leiden Comm., No. 133, p. 3.

${ }^{3}$ Leiden Comm., No. 139, pp. 65-71.
} 
In 1917 the suggestion was made 4 that the "critical current" and "critical magnetic field" were not independent phenomena, but that "the "threshold' value of current is that at which the magnetic field due to the current itself is equal to the critical magnetic field." At the time this hypothesis was published the experimental data available were not sufficient to afford a very convincing test of it.

Since that time a great deal of experimental work on supraconductivity has been done by Kamerlingh Onnes and his associates, and some of these results make possible a rather critical test of the hypothesis. In particular, two very clever experiments are described ${ }^{5,6}$ in which the specimen consists of a slender tube of tin which is influenced by the magnetic field both of the current flowing in the tin itself and of the current flowing in a separate wire which is placed along the axis of the tube. From a qualitative consideration of their results in these and other experiments Onnes and Tuyn concluded that "on the faith of the results obtained up till now, we think we may accept the hypothesis of Silsbee as being correct." 7

It seemed to the author that the careful experimental work at Leiden deserved a somewhat more detailed analysis than had been applied by the experimenters themselves and could give a more definite and quantitative test of the hypothesis. It is the purpose of the present paper to present such a detailed analysis and to compare the theoretical results thus obtained with the observed data.

\section{CALCULATIONS OF CURRENT DISTRIBUTION}

The essential element of these experiments is a system of conductors consisting of an outer hollow, circular cylinder of supraconducting material having its inner radius $a$ and outer radius $b$; and of a slender straight inner conductor, coaxial with the tube. Currents are established having values $I_{1}$ in the inner conductor and $I_{\mathrm{o}}$ in the outer cylinder. The problem is to find the electric potential gradient $E$ in the outer conductor, and incidentally the current density $i$ and magnetic field intensity $H$ at various points in the cross section of the cylinder.

A complete solution of the problem would involve a consideration of the time element and the working out of the theoretical history of the several variables throughout the course of the experiment. No such ambitious plan is here attempted. The following paragraphs will merely point out a particular set of steady-state current and flux distributions which has been found to be such as to satisfy the usual electromagnetic equations on the basis of the postulated assumptions.

\footnotetext{
- Note on Electrical Conduction in Metals at Low Temperatures, F. B. Silsbee, B. S. Sci. Paper No. 307; 1917.

${ }^{6}$ Weerstandsmetingen in Vloeibaar Helium, W. Tuyn, Leiden Diss.; 1924.

${ }^{6}$ The disturbance of supraconductivity by magnetic fields and currents, W. Tuyn and H. Kamerlingh Onnes, J. Franklin Inst., 201, p. 379; 1926.

7 J. Franklin Inst., 201, p. 407; 1926.
} 
The effect of persistent induced currents is considered briefly in a later paragraph.

To make the problem definite and as simple as possible the following assumptions will be made:

1. The material will be regarded as a continuous isotropic medium, free from Hall effect.

2. The currents, etc., will be considered constant in time; that is, transient phenomena will be disregarded.

3. The conductors will be assumed to be infinitely long so that end effects do not enter and the problem becomes two-dimensional only. From 2 and 3 it follows that the potential gradient, $E$, must be the same at all points throughout the volume of the outer cylinder.

4. The system will be considered to be symmetrical about its axis so that $H$ and $i$ become functions of the single variable $r$.

5. Maxwell's equation curl $H=4 \pi i$ is assumed to hold, and because of assumptions 3 and 4 it reduces to

$$
\frac{d(H r)}{d r}=4 \pi i r
$$

Integration of equation (1) shows that the total current $I$ flowing in the zone bounded by cylindrical surfaces of radii $r_{1}$ and $r_{2}$, respectively, is given by

$$
I=\frac{1}{2}\left(H_{2} r_{2}-H_{1} r_{1}\right)
$$

where $H_{2}$ and $H_{1}$ are the magnetic field intensities at the two boundary surfaces of the zone.

6. The material in the outer cylinder is assumed to have a definite transition magnetic field strength, $H_{\mathrm{c}}$, such that material located where the resultant magnetic field is less than $H_{\mathrm{c}}$ is supraconducting and has zero resistance, while material located where the field exceeds $H_{\mathrm{c}}$ has its normal resistivity and obeys Ohm's law.

7. For volume elements in which $H$ exactly equals $H_{c}$, the resistivity is indeterminate and may have any value between zero and the normal value. Hence, in such cases the value of the current density can not be calculated directly by applying Ohm's law, but must be deduced from other considerations.

From these assumptions it follows that there may exist in the cross section of the tubular conductor one or more annular zones of four different types. Each type of zone has its own type of field and current distribution and will be assigned a characteristic symbol as indicated in the following paragraphs:

1. Normal zone $(N)$.- - Here the material has normal resistivity, obeys Ohm's law, and hence the current density $i$ is uniform and given by

$$
i=E \lambda=\frac{E}{\pi R\left(b^{2}-a^{2}\right)}
$$


where $R$ is the normal resistance per unit length of the tube. Integration of equation (1) gives:

or

$$
\left.\begin{array}{r}
H=2 \pi E \lambda r+\frac{C}{r} \\
H=\frac{2 E r}{R\left(b^{2}-a^{2}\right)}+\frac{C}{r}
\end{array}\right\}
$$

and $H$ is by assumption 6 necessarily numerically greater than $\mathrm{H}_{\mathrm{c}}$. by

The total current $I$ in such a zone between radii $r_{1}$ and $r_{2}$ is given

$$
I=\frac{E\left(r_{2}^{2}-r_{1}^{2}\right)}{R\left(b^{2}-a^{2}\right)}
$$

2. Supraconducting zone (S).-By assumption 6 the magnetic field throughout such a zone must be between the limits $-H_{\mathrm{c}}$ and $+H_{\mathrm{c}}$ and also for any finite current density the potential gradient $E$ must be a small quantity of the same order as $\frac{R^{\prime}}{R}$ where $R^{\prime}$ is the possible "microresidual resistance" of the tube in the supraconducting state. A detailed determination of the current and flux distribution within the thickness of such zones is not required for the purpose of the present problem and is complicated by the possible existence of "persistent currents."

3. Thin supraconducting zone (s) or current sheet.-As the thickness of a supraconducting zone diminishes indefinitely the total current, $I$, in the zone is related to the difference $\Delta H$ in magnetic field intensity on the two sides of the zone by the equation

$$
I=\frac{\Delta H r_{0}}{2}
$$

where $r_{0}$ is the mean radius of the zone.

Since $\Delta H$ can not exceed $2 H_{\mathrm{c}}$, it follows that $I$ can not exceed $H_{\mathrm{c}} r_{\mathrm{o}}$. This means that any increase in $E$ which might tend to increase $I$ will merely cause a decrease in the thickness $\Delta r$ of the zone according to the relation

$$
E \Delta r=\frac{R^{\prime} \Delta H\left(b^{2}-a^{2}\right)}{4}
$$

where $R^{\prime}$ is as before the "microresidual resistance" per unit length.

4. Constant field zone $(C)^{8}$. - Within a certain range of possible combinations of currents in the inner and outer conductors a zone may exist such that, if the material in it had normal resistance, the magnetic field would be less than $H_{\mathrm{c}}$, yet if the material were supra-

8 The possibility of the existence of this type of zone was first suggested by Langevin in a letter to the author. 
conducting the resulting currents would produce a magnetic field greater than $H_{\mathbf{c}}$. Under such circumstances it seems reasonable to consider in accordance with assumption 7 that the current density would take on an intermediate value which would be just such as to maintain the magnetic field at a constant value, $H_{c}$, throughout the thickness of the zone.

In such a zone, Ohm's law leads to an indeterminate result for the current density. The fact that here we have $H=H_{\mathrm{c}}$ does however, in combination with the general equation (1) fix the current density as

$$
i=\frac{H_{\mathrm{c}}}{4 \pi r}
$$

The total current $I$ flowing in the shell between any two cylindrical surfaces of radii $r_{1}$ and $r_{2}$ is

$$
I=\frac{H_{\mathrm{c}}}{2}\left(r_{2}-r_{1}\right)
$$

The outer edge of such a zone may be either at the outer surface of the tube or at a radius $r_{2}$ such that

$$
r_{2}=\frac{H_{\mathrm{c}} R\left(b^{2}-a^{2}\right)}{4 E}=\frac{H_{\mathrm{c}}}{4 \pi E \lambda}
$$

beyond which lies a normal $(N)$ zone. This relation is necessitated by the fact that the current density at a radius greater than $r_{2}$ as fixed by equation (8) would be less than that produced by the gradient $E$ acting on the metal in the normal state.

Since the inner radius can not be less than $a$ the current density can not exceed the finite limit $\frac{H_{\mathrm{c}}}{4 \pi a}$.

The real physical relation corresponding to assumptions 6 and 7 is probably better expressed by stating that the resistivity changes continuously from normal at a field $H_{\mathrm{c}}+h$ to zero at a field $H_{\mathrm{c}}-h$, where the range $2 h$ within which the change of state occurs is relatively small. The corresponding description of a constant field zone would be one in which the field lies between the limits $H_{\mathrm{c}}+h$ and $H_{\mathrm{c}}-h$, while the current density in a corresponding way departs slightly from the value given by equation (8) and still physically is determined by the resistivity and potential gradient.

The number, type, and dimensions of the various zones which exist in the outer tube under any given set of conditions depend on the magnitudes of the currents $I_{1}$ and $I_{0}$. For any given temperature of the specimen $H_{\mathrm{c}}$ is fixed and any possible combination of $I_{1}$ and $I_{\mathrm{o}}$ corresponds to a point on Figure 1 in which $\frac{I_{0}}{b H_{c}}$ is plotted as abscissa and $\frac{I_{1}}{b H_{c}}$ as ordinate. The entire coordinate plane is seen to be 
divided up into regions. Within each region the arrangement of the concentric zones in the cross section of the tube is as indicated by the symbols $(N, N s C N$, etc.) marked on the region. The boundaries of the regions are the loci of combinations of $I_{1}$ and $I_{0}$ for which one

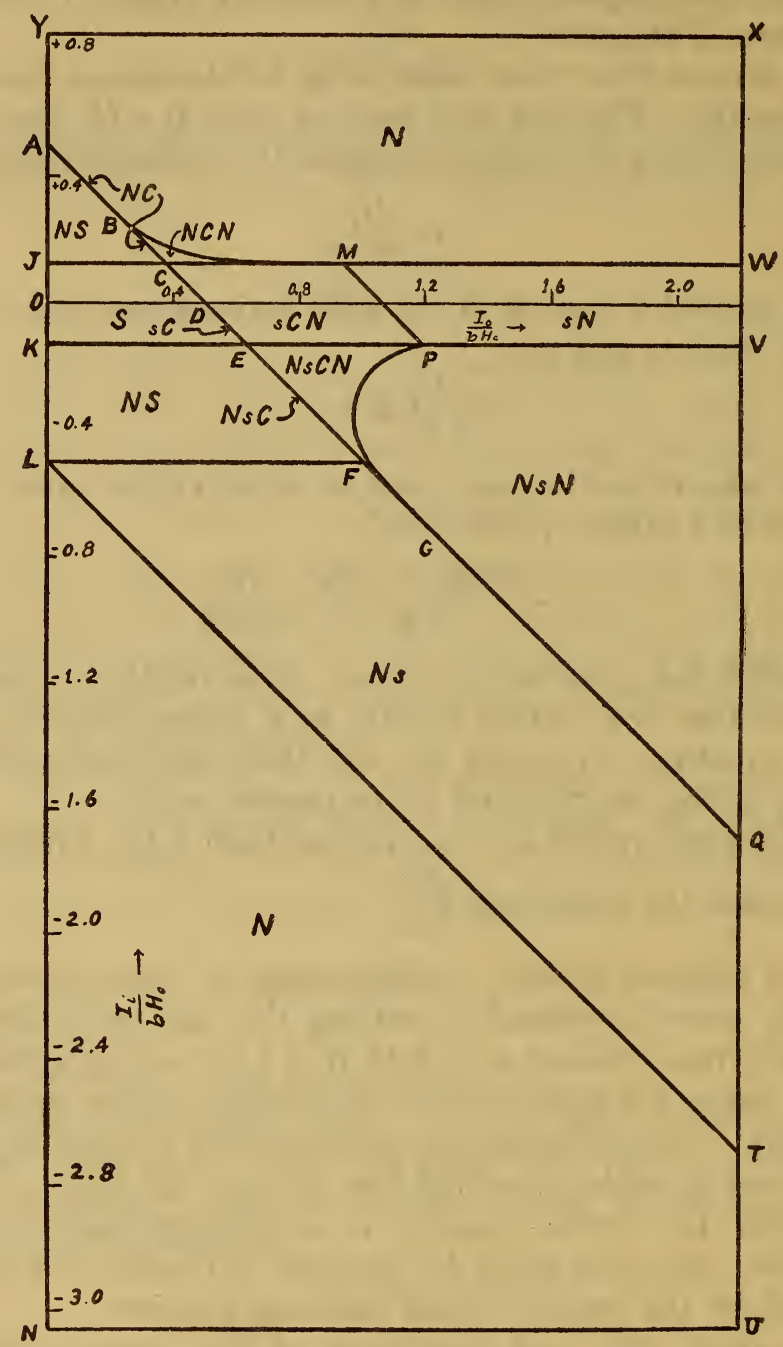

Frg. 1.-Map of the $I_{o}, I_{i}$ surface for the case of $a=0.25 b$, showing boundaries between regions having various combinations of zones

zone is just at the point of appearing or disappearing from the cross section. For all points within a given region the order and type of the zones is the same although the radii of the zones may vary. In each symbol the zones are given in order of increasing radius. Thus " $N s C N$ " indicates the presence of a normal $(N)$ zone next the inner 
wall of the tube. This is surrounded in turn by a thin supraconducting sheet $(s)$ and by a constant field zone $C$, while another normal zone $N$ lies outside of the combination and next to the outer surface of the conductor. The location of these several regions on the $I_{1}, I_{0}$ plane involves a rather long, though simple process of analysis. A summary of this will be found in the appendix.

We may regard the potential gradient $E$, which is directly measured in the experiments as the dependent variable which is fixed by the current values $I_{1}$ and $I_{0}$; and construct a surface in space by plotting $I_{1}$ and $I_{0}$ as rectangular coordinates and erecting perpendicular to their plane at each point a coordinate proportional to the corre-

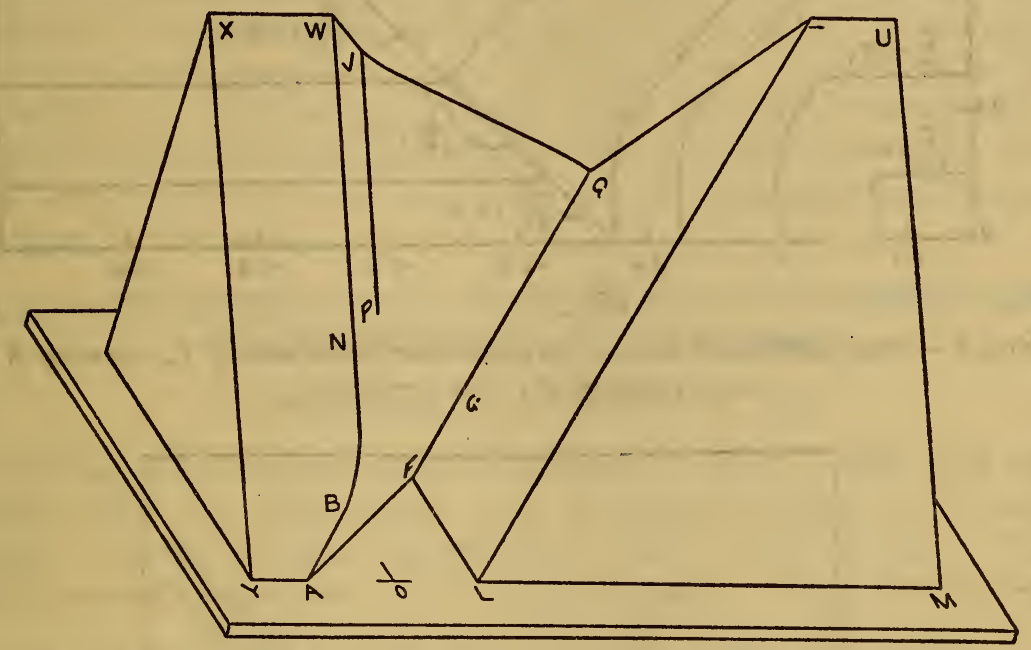

Fig. 2.-Projective view of the $E, I_{o}, I_{i}$ surface, constructed for $a=0.25 b$

sponding value of $E$. Figure 2 shows such a surface drawn in projection for the case of $a=0.25 b$.

Figure 3 shows a series of typical cross sections of this surface taken for a number of constant values of $I_{0}$, and hence showing $E$ as a function of $I_{1}$. Similarly Figure 4 shows cross sections for constant $I_{1}$ and hence showing $E$ as a function of $I_{0}$.

In the foregoing discussion no account has been taken of the possible existence of eddy currents induced at the time the various currents were established and persisting thereafter. It seems from the following relations, however, that such currents could not materially affect the distribution described above.

Any persistent current can exist only in the supraconducting material and can not flow in the resisting leads outside the cryostat. Hence by assumption 4 it must flow in opposite directions in filaments which lie in two concentric cylindrical surfaces so that the $49649^{\circ}-27-2$ 


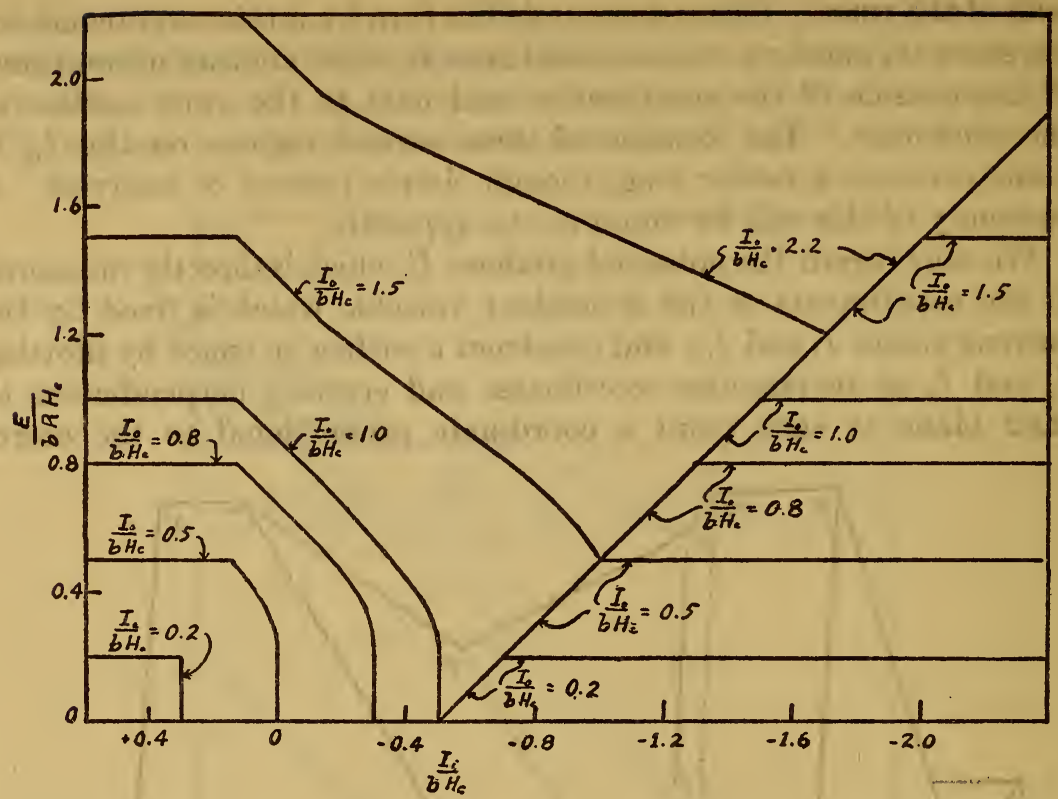

Frg. 3.-Cross sections of surface perpendicular to the axis of $I_{o}$, showing $E$ as a function of $I_{i}($ for $a=0.25 b)$

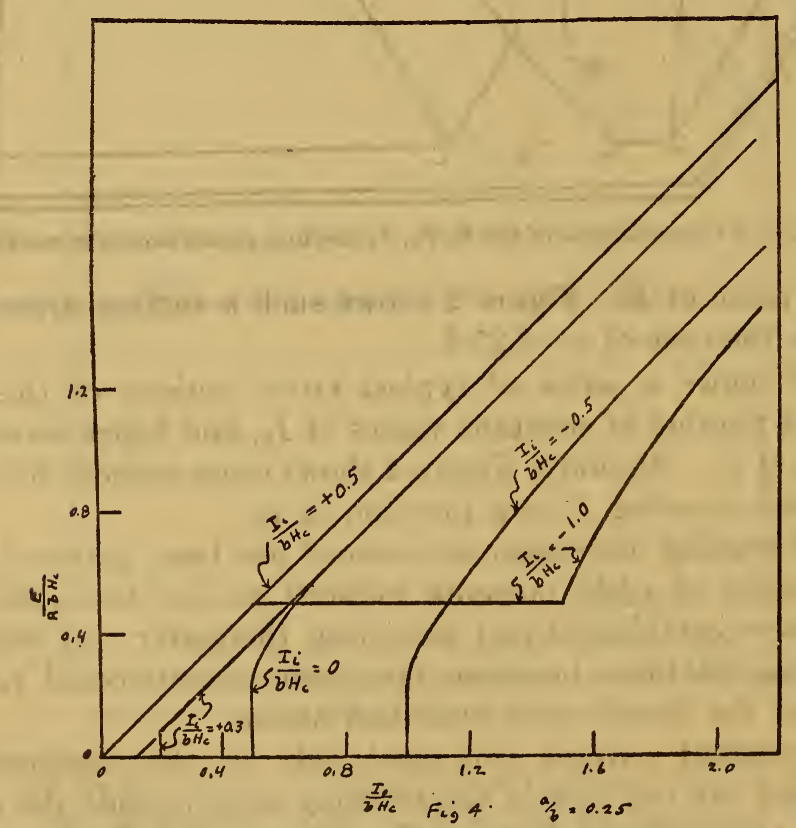

Fig. 4.-Cross sections of surface perpendicalar to the axis of $I_{i}$, showing $E$ as a function of $I_{0}$ (for $\left.a=0.25 b\right)$ 
total eddy current integrates to zero over the entire cross section. The currents in each elementary pair of such surfaces will exert no magnetic field at points outside or inside of both, but will tend to shield the space between the two surfaces. Now, none of the distributions found above contains more than one supraconducting zone. Hence the effect of persistent currents will be only that of modifying the distribution of current density and field inside the $S$ zone and will not affect the existence of, nor the current, nor field in other zones.

In the deduction of the arrangements of zones by means of Figure 8 in the appendix it has been found unnecessary to specify the exact current distribution within an $S$ zone. Any set of eddy currents may, therefore, be present as a result of the previous history of the specimen without affecting the conclusions here given. It seems probable ${ }^{9}$, provided the specimen was cooled below the critical temperature before the currents were established, that the current even in what has been called an $S$ zone really resides in thin sheets at the bounding surfaces of the zone.

\section{COMPARISON WITH EXPERIMENTS OF ONNES AND TUYN ON TUBULAR SPECIMENS}

\section{EXPERIMENTS OF MARCH 12, $1924^{10}$}

During these experiments the currents $I_{1}$ and $I_{0}$ were held constant while the temperature was decreased through the critical range. During the first experiment $I_{1}=0, I_{0}=2.50$ amperes. During the second experiment $I_{0}=I_{1}=2.50$ amperes. The outer tube was of tin $10 \mathrm{~cm}$ long, $b=0.25 \mathrm{~cm}, a=0.240_{6}$. Hence $\frac{a}{b}=0.963$.

Since $H_{\mathrm{c}}$ is a function of temperature, and since the coordinates of Figures 1 and 6 are the quantities $\frac{I_{0}}{b H_{\mathrm{c}}}$ and $\frac{I_{1}}{b H_{\mathrm{c}}}$, it follows that the variation of temperature used in the experiments was equivalent to a continuous though not necessarily linear variation of the coordinates of these figures. In the first experiment the variation was along a line such as $O Q$, Figure 6, while in the second it was along a line as $O P$. Now for the $s N$ region the potential gradient as given by equation (23) (see appendix) for $I_{0}=0$ reduces to

$$
E_{1}=R\left(I_{0}-\frac{a H_{\mathrm{c}}}{2}\right)
$$

where $R=\frac{1}{\pi \lambda\left(b^{2}-a^{2}\right)}$ is the normal resistance of the tube per unit length.

\footnotetext{
- See G. Breit, Transients of Magnetic Field in Supraconductors. Proc. K. Ak\&d. v. Wetensch., Amsterdam, 26, p. 529; 1923.

10 See footnotes 5 and 6, p. 294.
} 
Similarly for the $N s$ region used in the second part of the experiment, equation (20a) gives

$$
E_{2}=R\left(-I_{1}-\frac{H_{\mathrm{c}} b}{2}\right)
$$

For the present case where $I_{1}=-I_{0}$, the difference in these quantities is

$$
E_{1}-E_{2}=\frac{R H_{\mathrm{c}}}{2}(b-a)
$$

For high temperatures where $H_{\mathrm{c}}$ is small this difference vanishes and for temperatures so low that $H_{\mathrm{c}}=\frac{2 I_{\mathrm{o}}}{b}$ (which is where the vertical $s C$ region occurs)

$$
E_{1}-E_{2}=R I_{\mathrm{o}} \frac{(b-a)}{a}=0.039 R I_{\mathrm{o}}
$$

for the specimen used.

We thus see that relatively little difference is to be expected between the results of the two experiments. In Figure 5 there are

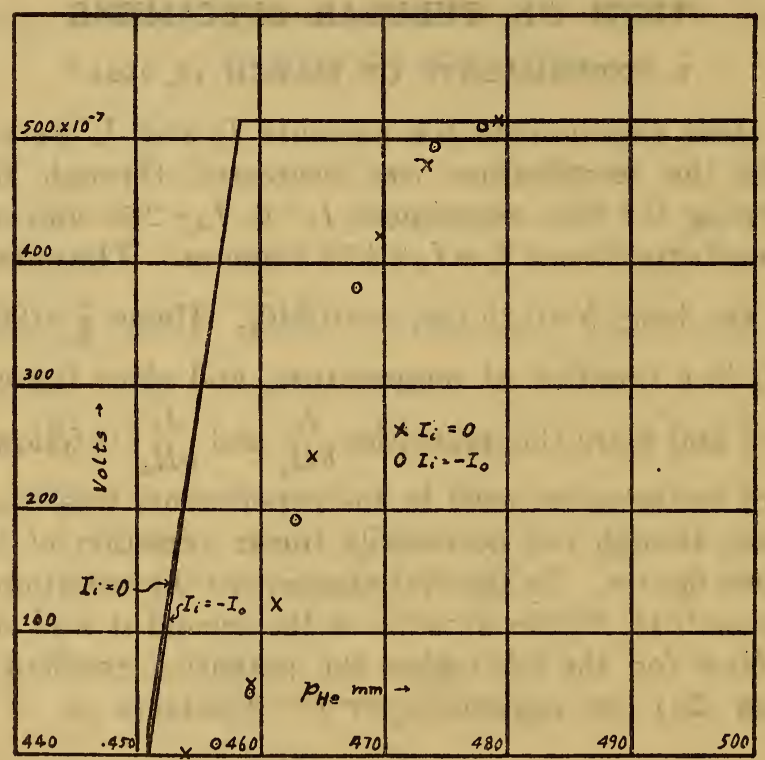

Fig. 5.-Voltage across tube as a function of temperature (expressed in terms of the vapor pressure of helium $P_{\mathrm{he}}$ ) The points were obtained by the experiments of March 12, 1924. The lines show the theoretical relations.

plotted as crosses the results of the first $\left(I_{1}=0\right)$ experiment, and as circles, the second $\left(I_{1}=-I_{0}\right)$ experiment. The solid lines show the values as computed from the foregoing equations, on the assumption 
that the normal resistance of the tube is $R=\frac{515 \times 10^{-7}}{2.5}$ ohms which corresponds to the highest observed voltage, and that the relation between $H_{c}$ and temperature is that given by the equation in Tuyn's Leiden Dissertation (p. 93)

$$
H_{\mathrm{c}}=-20.3 T^{2}+281.4 \text { gauss }
$$

This equation is based on data obtained over a considerable range of temperature, 1.5 to $3.74^{\circ} \mathrm{K}$., and hence may not be exactly correct at the extreme end of this range where it is here applied. A change of 1.3 in the last term of equation (14) or of 0.1 in the coefficient of $T^{2}$ would be sufficient to make the theoretical curves pass through the center of the plotted points. The more gradual slope of the observed curve would be accounted for very plausibly if the variation of resistance with field or temperature were not quite as abrupt as has here been assumed.

\section{EXPERIMENTS OF APRIL 17, 1924}

In each of these experiments both the temperature (and hence $H_{\mathrm{c}}$ ) and the current $I_{\mathrm{o}}$ were held constant while $I_{1}$ was varied. Three experiments were made at temperatures of $3.66,3.67$ and $3.69^{\circ}$ $\mathrm{K}$., respectively. In all cases $I_{0}$ was 2.0 amperes. The specimen used in these experiments had $a=0.0307, b=0.040, e=10 \mathrm{~cm}$, and hence $a=0.75 b$. Figure 6 is a map of the $E, I_{1}, I_{0}$ surface for a tube of these proportions. The values of voltage to be expected correspond to a section of the theoretical surface perpendicular to the $I_{0}$ axis along the lines $A B, C D$, and $E F$, respectively.

A number of such sections are shown in Figure 3 for various values of $I_{\mathrm{o}} / b H_{\mathrm{c}}$ for the case of $a=0.25 b$. On comparing these with Figure $7^{10}$ of Tuyn and Onnes, it will be noted that the observed curves agree with the theoretical in the following qualitative features:

(a) Lower values of $E$ at $I_{1}=0$ than at large values of $I_{1}$.

(b) A flat $U$ curve at low temperatures (high $H_{c}$ ).

(c) A sharp $\mathrm{V}$ curve at higher temperature (low $H_{\mathrm{c}}$ ).

(d) A flattening out at high values of $I_{1}$.

Figure 7 shows the theoretical curves plotted to the same coordinates as the observed points for the particular conditions of the experiments of April 17, 1924. In this plot the values of $H_{\mathrm{c}}$ were obtained from equation (14) and the normal resistance of the specimen was taken as $R=\frac{2518 \times 10^{-7}}{2.0} \mathrm{ohms}$, thus forcing an agreement at the last point plotted. It will be seen that the agreement is on the whole fairly good, especially as regards the slope and relative position of the lines

10 J. Frank. Inst., 201. p. 408; A pril, 1926. 
on the right-hand side of the minimum. The differences are, however, systematic and are roughly equivalent to a shift of 0.5 ampere. The cause of these differences must apparently be sought in a failure of the assumption of the sudden discontinuity in resistance, or in minor experimental imperfections, such as slight eccentricity of the

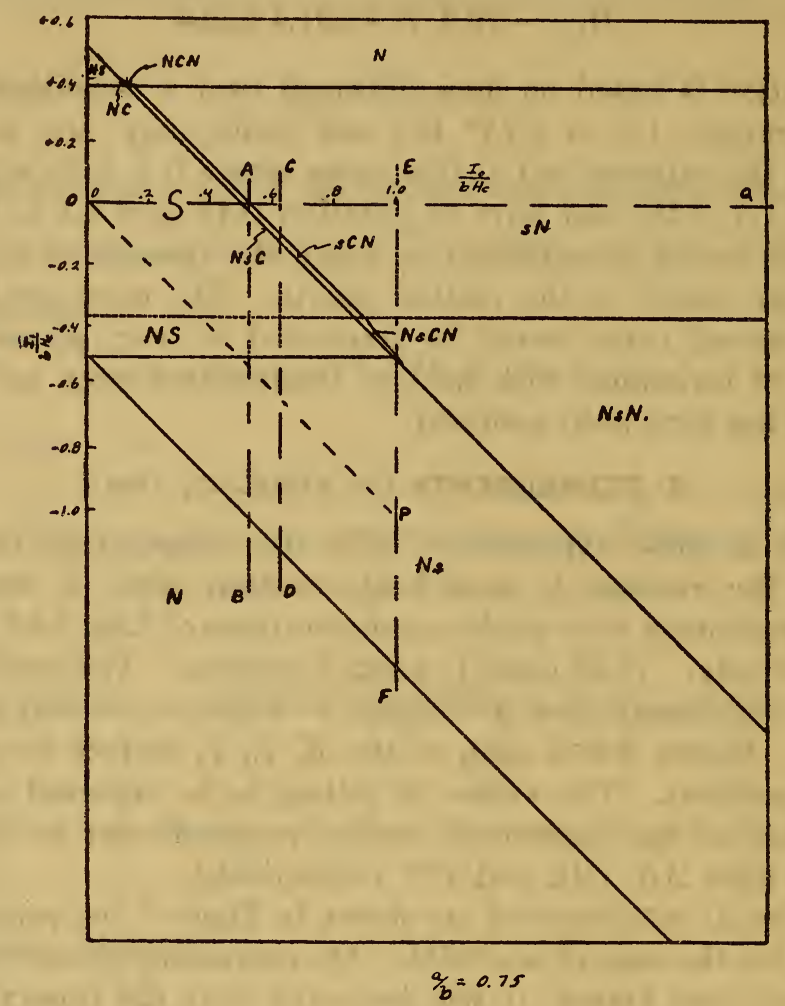

Fig. 6. - Map of the $I_{o}, I_{i}$ surface for the case of $a=0.75 \mathrm{~b}$, showing the boundaries between regions having various combinations of zones

inner conductor, magnetic field from return leads and earth, end effects, heating effects, etc.

\section{CONCLUSIONS}

In a certain sense the hypothesis of the correspondence of "critical currents" and "critical field" can not be established by a crucial experiment. The hypothesis is equivalent to the statement that a critical current will not be found except under conditions where its magnetic field is sufficient to account for the restoration of resistance. Any single experiment which did demonstrate such a saturation current in the absence of appreciable magnetic field would, indeed be a crucial disproof of the hypothesis. On the other hand, any number 
of experiments like the present ones, in which an apparent "critical current" can be accounted for by the magnetic field, can not suffice to prove the hypothesis for all possible cases.

However, we have here data obtained with a distribution of magnetic field closely similar to that in any conductor carrying

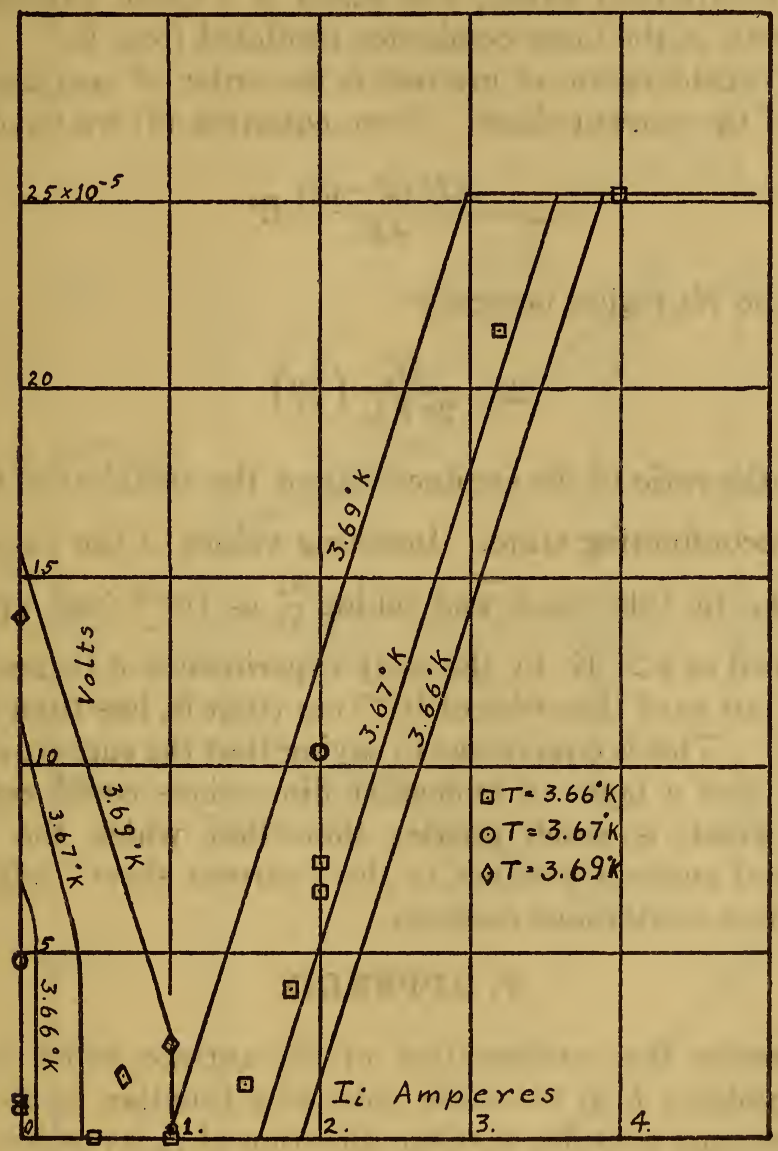

Fig. 7.-Voltage across tube as a function of $I_{i}$, for $I_{0}=2.0$ amperes and three different temperatures

The points were obtained by theiexperiments of April 17, 1924. The lines show the theoretical relations.

a current and find that the results can be completely explained without making use of the concept of critical current.

In fact a resistance which apparently had been produced by giving $I_{0}$ a value in excess of the "critical current." was found to be neutralized by the purely magnetic effect of $I_{1}$. Such a neutralization would be most improbable if the "critical current" were the result of some radically different effect, such for instance as a Peltier heating at crystal junctions. 
Some by-products of the foregoing discussion are worthy of mention and, perhaps, of experimental study. One of these is the curious properties of the conductor system when in the $N s$ region. Here according to the preceding equations we should have a steady potential gradient which is independent of the current flowing in the conductor in which the gradient exists, but which is a linear function of the steady current in the inner conductor insulated from it.

Another consideration of interest is the order of magnitude of the thickness of the current sheet. From equation (6) we have

$$
\Delta r=\frac{\Delta H\left(b^{2}-a^{2}\right)}{4 E} R^{\prime}
$$

which for the $N s$ region becomes

$$
\Delta r=\frac{H_{\mathrm{c}}}{2 \pi E \lambda_{\mathrm{o}}}\left(\frac{\lambda_{\mathrm{o}}}{\lambda^{1}}\right)
$$

where $\frac{\lambda_{0}}{\lambda^{1}}$ is the ratio of the conductivity of the metal at $0^{\circ} \mathrm{C}$. to that in the supraconducting state. Inserting values of the various quantities for the tin tube used, and taking $\frac{\lambda_{0}}{\lambda^{1}}$ as $10^{-10}$ (the upper limit found for lead at $4.2^{\circ} \mathrm{K}$. by the early experiments of Onnes), we find $\Delta r$ to come out as of the order of $10^{-10} \mathrm{~cm}$ (that is, less than molecular dimensions). This is equivalent to saying that the supraconductivity is so great that a layer of molecular dimensions could easily carry a current which is much greater than that which the foregoing mathematical analysis ascribes to the "current sheet" which would be present in a continuous medium.

\section{APPENDIX}

To determine the configuration of the surface which shows the potential gradient $E$ in the outer tube as a function of the currents $I_{1}$ and $I_{0}$, we may consider that the direction of $I_{0}$ is positive and that the magnetic field produced by it alone is positive. The sign of $I_{1}$ will be + or - according as it flows in the same or the opposite direction as compared to $I_{0}$.

The succession of types of zone which are possible in the various cases can best be seen from a consideration of Figure 8. Here the quantity $\mathrm{Hr}$ has been plotted as ordinate against $r$ as abscissa. The straight lines $D N$ and $F S$ are drawn through the origin $O$ with slopes of $+H_{\mathrm{c}}$ and $-H_{\mathrm{c}}$, respectively. The abscissa $O E$ corresponds to the inner radius $a$ and $O Q$ to $b$. The distribution of the quantity $(H r)$ over the cross section of the tube under any condition is represented on this graph by a curve which extends from the line $A J$ to 


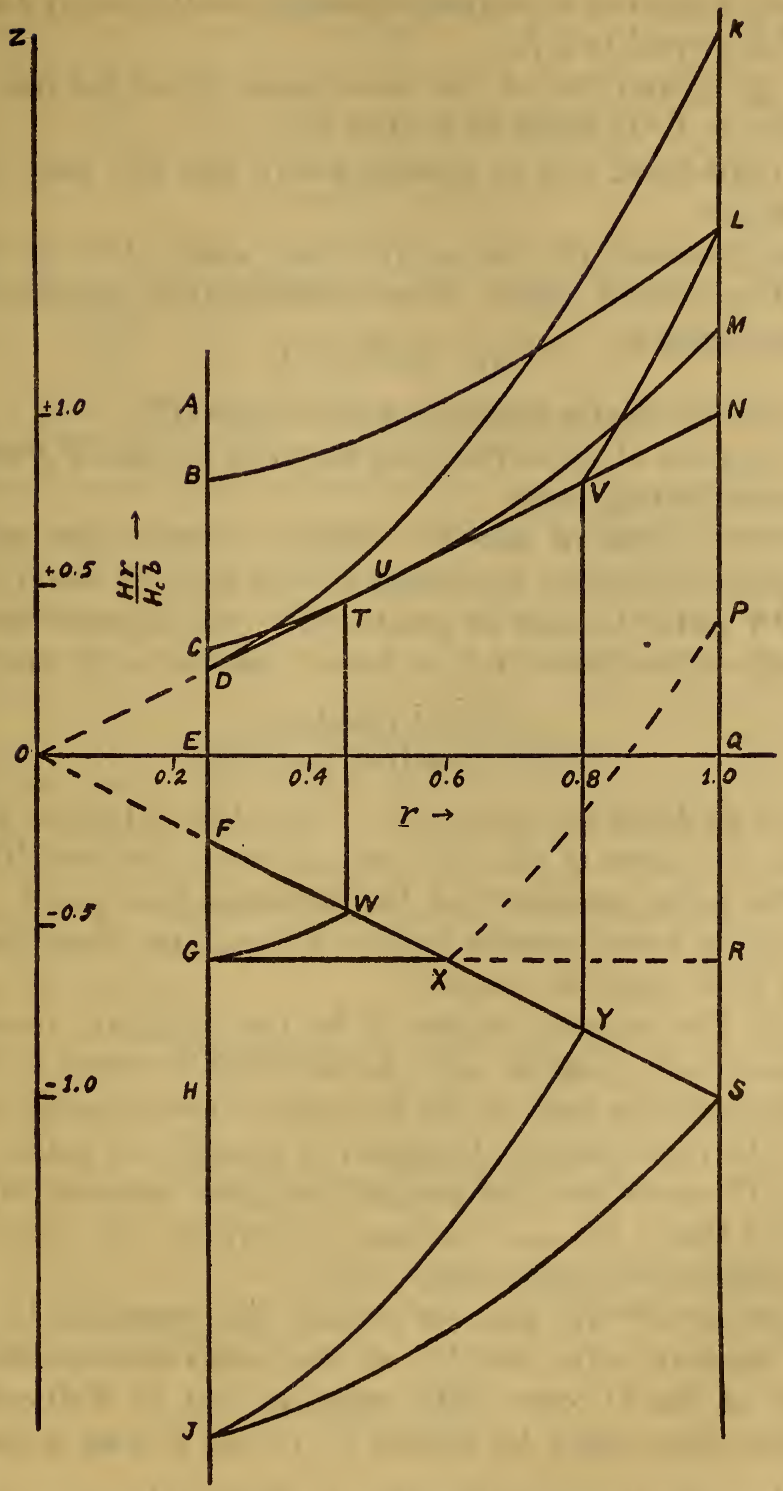

FIG. 8.-A graph showing the quantity $\mathrm{Hr}$ in the outer cylinder plotted against $r$, for various possible combinations of currents

The coordinates are expressed in terms of the fixed quantities $\mathrm{H}_{0} \mathrm{~b}$ and $b$ as units. 
the line $K S$ and which also satisfies a number of other conditions as follows:

1. The left-hand end of the curve must be on the line $A J$ at a height (such as $B E$ ) equal to $2 I_{1}$.

2. The right-hand end of the curve must lie on the line $K S$ at a height (such as $L \cap$ ) equal to $2\left(I_{1}+I_{0}\right)$.

3. The right-hand end is always above the left end, since $I_{0}$ is always positive.

4. Those portions of the curve lying above $D N$ or below $F S$ correspond to normal zones. Consequently from equation (4) they satisfy the equation

$$
H r=2 \pi E \lambda r^{2}+C
$$

and hence are parts of a parabola with its axis $O Z$.

5. That portion of the curve lying between $D N$ and $F S$ corresponds to a supraconducting zone.

6. Since the slopes of the $H r$ versus $r$ curves are proportional to the current densities, the slope of the part of the curve lying between $D N$ and $F S$ must be greater than the slopes of the portions lying outside these limits by a factor which is of the order of

\section{$\frac{\text { Normal resistance }}{\text { Micro residual resistance }}$}

and hence very large (at least $10^{8}$ ). From this it follows that

7. When the slope of the $H r$ curve between the lines $D N$ and $F S$ is finite, the parts outside must be horizontal lines; and

8. When the parts outside have a finite slope, then the part between the lines must be vertical.

9. Unless the current density is in the negative direction (and this is conceivably possible only in the eddy currents in the supraconducting zone) the slope of the $H r$ curve is always positive. Hence

10. The $H r$ curve can cut the line $F S$ at only one point.

11. The Hr curve may for part of its course coincide with the line $D N$. When this is the case the zone over which the curves coincide has the constant magnetic field, $+H_{\mathbf{c}}$.

12. When an $H=H_{\mathrm{c}}$ zone is present the parabola in the outer $N$ zone is tangent to the line $D N$ at the radius corresponding to the outer edge of the $C$ zone. This relation may be deduced from the fact that the outer edge (of radius $r_{1}$ ) of the $C$ zone is fixed by the relation that the current density at that radius, viz, $\frac{H_{c}}{4 \pi r_{1}}$ is the same as the density $E \lambda$ in the adjacent $N$ zone. If the density were greater, the radius would be within the $C$ zone, while the density can not be less since the applied potential gradient can produce a 
current density $E \lambda$ even in normal material. Inserting this value of $E \lambda$ into equation (1a) gives

or, since

$$
H r=\frac{H_{\mathrm{c}}}{2 r_{1}} r^{2}+C
$$

$$
\begin{gathered}
H=+H_{\mathrm{c}} \text { at } r=r_{1} \text {, so that } C=\frac{H_{\mathrm{c}} r_{1}}{2} \\
H r=\frac{H_{\mathrm{c}} r^{2}}{2 r_{1}}+\frac{H_{\mathrm{c}} r_{1}}{2}
\end{gathered}
$$

as the equation of the parabola in the outer $N$ zone. The slope of this curve is $\frac{d(H r)}{d r}=\frac{H_{\mathrm{c}} r}{r_{1}}$ and at $r=r_{1}$ both the ordinate and the slope of the parabola become the same (viz, $H_{c}$ ) as those of the line $D N$. These are the conditions for tangency.

An inspection of Figure 8 shows that the foregoing 12 conditions are sufficient to locate the $H r$ curve without ambiguity, except where it crosses the supraconducting region between $D N$ and $F S$. Even here its location is fixed when the potential gradient has a finite value for the supraconducting zone must then degenerate to a current sheet, and the $\mathrm{Hr}$ curve to a vertical line. The number of different possible cases which require separate discussion is, however, rather large since the curve may start at any point on the line $A J$ and end at any higher point on the line $K S$, and each crossing of the limiting lines $F S$ and $D N$ introduces a zone boundary.

As an example we may take a case such as curve $G W T U M$, Figure 8, which corresponds to the $N s C N$ region of Figure 1. For this case at the outside

$$
H_{\mathrm{b}} b=2\left(I_{1}+I_{0}\right)=\frac{H_{\mathrm{c}} b^{2}}{2 r_{1}}+\frac{H_{\mathrm{c}} r_{1}}{2}
$$

whence

$$
r_{1}=\frac{2\left(I_{1}+I_{0}\right)}{H_{\mathrm{c}}}-\sqrt{\frac{4\left(I_{1}+I_{\mathrm{o}}\right)^{2}}{H_{\mathrm{c}}^{2}}-b^{2}}
$$

The inner radius of the $C$ zone $r_{\mathrm{o}}$ is given by the fact that a parabola similar to $U M$ (equation $2 \mathrm{a}$ ), but with a different valuo of $C$ must pass through point $G$, hence

and at $W$

$$
C^{\prime}=2 I_{1}-\frac{H_{\mathrm{c}} a^{2}}{2 r_{1}}
$$

$$
-H_{\mathrm{c}} r_{\mathrm{o}}=2 I_{1}+\frac{H_{\mathrm{c}}}{2 r_{1}}\left(r_{\mathrm{o}}^{2}-a^{2}\right)
$$

The potential gradient is given directly by

$$
E=\frac{H_{\mathrm{c}}}{4 \pi r_{1} \lambda}=\frac{H_{\mathrm{c}} R\left(b^{2}-a^{2}\right)}{4 r_{1}}
$$


where $R$ is the total resistance per unit length of the outer tube in the normal state.

It will be noted from equation (5a) that $r_{1}$ depends only on the quantity $\left(I_{1}+I_{0}\right)$; that is, on the outer end point of the Hr curve. Consequently equations (5a) and (7a) apply for any location of the beginning point for which the $C$ zone exists. These equations, therefore, apply also to the $N C N$ and $s C N$ regions of Figure 1.

The boundaries of this group of regions are located as follows:

As $I_{1}+I_{0}$ is decreased, $M$ (in fig. 8) moves down and the foregoing. relations apply until $I_{1}+I_{0}=\frac{b H_{\mathrm{c}}}{2}$. Then $M$ is at $N$ and $r_{1}=b$ (by equation 5a). The regions are thus bounded by the line $B G$ (fig. 1 ). If $\frac{a H_{\mathrm{c}}}{2}>I_{1}>-\frac{a H_{\mathrm{c}}}{2}$, the beginning of the curve must lie between $D$ and $F$ (fig. 8) and the $C$ region will vanish if $I_{1}+I_{0}$ is increased enough to make $r_{1}=a$. This occurs when

$$
I_{1}+I_{\mathrm{o}}=\frac{H_{\mathrm{c}}\left(b^{2}+a^{2}\right)}{4 a}
$$

This corresponds to the line $M P$ (fig. 1), and beyond this line an $s N$ region must exist. At point $M, I_{0}=\frac{H_{\mathrm{c}}\left(b^{2}-a^{2}\right)}{4 a}$ and at $P$ along the line $M P$

$$
I_{0}=\frac{H_{\mathrm{c}}\left(b^{2}+3 a^{2}\right)}{4 a}
$$

$$
E=\frac{H_{\mathrm{c}} R\left(b^{2}-a^{2}\right)}{4 a}
$$

If $I_{1}>\frac{a H_{\mathrm{c}}}{2}$ the $C$ zone may vanish if the beginning point (as $C$ ) is higher than that at which a parabola through the end point (as $M$ ) tangent to $D N$ would cut $A J$. Such a parabola is defined by equation (3a) and its intercept with $A J$ is given by

$$
2 I_{1}=\frac{H_{\mathrm{c}}\left(a^{2}+r_{1}^{2}\right)}{2 r_{1}}
$$

where $r_{1}$ is given by equation (5a). Inserting this in equation (4a) gives

$$
2 I_{\mathrm{o}}=\frac{H_{\mathrm{c}}\left(b^{2}-a^{2}\right)}{2 r_{1}}
$$

as the other parametric equation for the boundary $B M$ (fig. 1). At $B, I_{1}=\frac{H_{\mathrm{c}}\left(b^{2}+a^{2}\right)}{4 b}$ and $I_{0}=\frac{H_{\mathrm{c}}\left(b^{2}-a^{2}\right)}{4 b}$. Beyond this boundary the tube is normal over the entire cross section. 
If $I_{1}<-\frac{a H_{\mathrm{c}}}{2}$ the $C$ zone will vanish in a similar fashion when $r_{\mathrm{o}}$ as given by equation (6a) is equal to $r_{1}$ as given by equation (5a). A direct solution for the locus ( $P G$, fig. 1 ) in terms of $I_{1}$ and $I_{0}$ leads to very complicated expressions. It can, however, be expressed quite simply in terms of $r_{1}$ or $r_{0}$ as a parameter by

$$
I_{1}+I_{0}=\frac{H_{\mathrm{c}}}{4 r_{\mathrm{o}}}\left(b^{2}+r_{\mathrm{o}}^{2}\right)
$$

and

$$
I_{1}=-\frac{H_{\mathrm{c}}}{4 r_{\mathrm{o}}}\left(3 r_{\mathrm{o}}^{2}-a^{2}\right)
$$

These equations result directly from equations (3a) and (6a). At $G, r_{0}=b$ and hence $I_{1}=-\frac{H_{\mathrm{c}}\left(3 b^{2}-a^{2}\right)}{4 b}$ and $I_{\mathrm{o}}=\frac{H_{\mathrm{c}}\left(5 b^{2}-a^{2}\right)}{4 b}$. Beyond this boundary lies an $N s N$ region.

The $N s N$ region corresponding to curves such as $J Y V L$ (fig. 8) can be treated in a similar way.

For the inner $N$ zone equation (1a) gives at $r=\mathrm{a}$

$$
2 I_{1}=2 \pi E \lambda a^{2}+C
$$

whence

$$
C=2 I_{1}-2 \pi E \lambda a^{2}
$$

then at point $Y$ the radius of which is $\mathrm{r}_{\mathrm{o}}$

Similarly at point $V$

$$
-H_{\mathrm{c}} r_{\mathrm{o}}=2 \pi E \lambda\left(r_{\mathrm{o}}^{2}-a^{2}\right)+2 I_{1}
$$

whence

$$
+H_{\mathrm{c}} r_{\mathrm{o}}=2 \pi E \lambda\left(r_{\mathrm{o}}^{2}-b^{2}\right)+2\left(I_{1}+I_{0}\right)
$$

$$
I_{\mathrm{o}}=\pi E \lambda\left(b^{2}-a^{2}\right)+H_{\mathrm{c}} r_{\mathrm{o}}
$$

Equations (14a) and (16a) furnish a solution for $E$ in terms of $r_{0}$ as a parameter, and conversely. The region is bounded at the top by the line $P V$ (fig. 1) for which $I_{1}=-\frac{H_{\mathrm{c}} a}{2}$ and along which $r_{\mathrm{o}}=a$ and

$$
E=\frac{I_{0}-H_{\mathrm{c}} a}{\pi \lambda\left(b^{2}-a^{2}\right)}
$$

The slope of the surface changes abruptly at this boundary. The lower boundary $G Q$ is where $r_{0}=b$ and by equation (15a)

while

$$
I_{1}+I_{\mathrm{o}}=\frac{H_{\mathrm{c}} b}{2}
$$

$$
E=\frac{-I_{1}-\frac{H_{\mathrm{c}} b}{2}}{\pi \lambda\left(b^{2}-a^{2}\right)}
$$


Along the curve $P G$ the region is bounded by the $N_{s} C N$ region and an examination of the derivatives shows that there is no discontinuity in the slope of the surface on passing this boundary.

When $\frac{-b H_{0}}{2}<I_{1}+I_{0}<+\frac{b H_{0}}{2}$, the end of the $H r$ curve lies at some point such as $P$ between $N$ and $S$. If the initial point lies between $D$ and $F$ (that is, $\left.-\frac{a H_{\mathrm{c}}}{2}<I_{1}<\frac{+a H_{\mathrm{c}}}{2}\right)$ the entire cross section is supraconducting and we have an $S$ region and $E$ is vanishingly small.

If the initial point lies at some point $G$ between $F$ and $H$ (that is, if $\frac{-b H_{\mathrm{c}}}{2}<I_{1}<\frac{-a H_{\mathrm{c}}}{2}$ ), the $H r$ curve must be a horizontal line from $G$ to $X$, where it enters the supraconducting region. Since the latter is of finite width, $E$ must still be zero. The radius of point $X$ is $\frac{-H_{\mathrm{c}}}{2 I_{1}}$.

If the initial point lies below $H$, as for instance at $J$, the $H r$ curve must have a vertical trend as $J S P$ and correspond to an $N s$ region. For such a curve equation (1a) becomes

from which

$$
2 I_{1}=2 \pi E \lambda a^{2}+C
$$

and

$$
C=2 I_{1}-2 \pi E \lambda a^{2}
$$

$$
H r=2 \pi E \lambda\left(r^{2}-a^{2}\right)+2 I_{1}
$$

at $S$

$$
-H_{\mathrm{c}} b=2 \pi E \lambda\left(b^{2}-a^{2}\right)+2 I_{1}
$$

or

$$
E=\frac{-I_{1}-\frac{H_{\mathrm{c}} b}{2}}{\pi \lambda\left(b^{2}-a^{2}\right)}
$$

and is not dependent on $I_{0}$. This part of the surface is therefore a plane sloping upward with increasing $I_{\mathrm{i}}$, but with its trace on the $I_{1}, I_{\mathrm{o}}$ plane parallel to the axis of $I_{0}$. For $\left(I_{1}+I_{\mathrm{o}}\right)<-\frac{b H_{\mathrm{o}}}{2}$ the entire curve must lie below the line $F S$ and the material is normal, so that

When

$$
E=\frac{I_{\circ}}{\pi \lambda\left(b^{2}-a^{2}\right)}=R I_{0}
$$

$$
\frac{-a H_{\mathrm{c}}}{2}<I_{1}<\frac{+a H_{\mathrm{c}}}{2} \text { but } I_{1}+I_{\mathrm{o}}>\frac{H c\left(b^{2}+a^{2}\right)}{4 a}
$$

(see equation (8a) it is evident from Figure 8 that the outer end of the $\mathrm{Hr}$ curve (as $E D K$ ) must lie in normal material and have a finite slope. The portion of the curve in the supraconducting 
region must, therefore, be vertical from its initial point (as $E$ ) up to $D$ and an $s N$ region results. The constant $C$ in equation (1a) can be determined from point $K$ as $2\left(I_{1}+I_{0}\right)-2 \pi E \lambda b^{2}$, hence the equation becomes

$$
H r=2 \pi E \lambda\left(r^{2}-b^{2}\right)+2\left(I_{1}+I_{0}\right)
$$

and since the curve passes through point $D$, we have

$$
E=\frac{I_{\mathrm{o}}+I_{1}-\frac{a H_{\mathrm{c}}}{2}}{\pi \lambda\left(b^{2}-a^{2}\right)}=R\left(I_{1}+I_{1}-\frac{a H_{\mathrm{c}}}{2}\right)
$$

The locus $A G$ (fig. 1) corresponding to $I_{1}+I_{0}=\frac{+b H_{\mathrm{c}}}{2}$ is marked by a discontinuity in $E$. Over the $S$ and $N S$ regions below this line the potential gradient is zero. Above this line, however, there exist the $N C N, s C N$, and $N s C N$ regions at the edge of which the potential gradient has the finite value, $\frac{H_{\mathrm{c}}}{4 \pi b \lambda}$. It is, however, quite possible that the experimental arrangement might apply to the specimen a potential gradient intermediate between these values while the current had exactly the value $I_{0}=\frac{b H_{\mathrm{c}}}{2}-I_{1}$. Such a condition would exist, for example, if a battery of voltage $E_{\mathrm{B}}$ in series with a rheostat of resistance $R_{\mathrm{e}}$ was connected to the tube of length $l$. We would then have the potential gradient given by

$$
E=\frac{1}{l}\left(E_{\mathrm{b}}-R_{\mathrm{e}} I_{\mathrm{o}}\right)
$$

or on inserting the value of $I_{0}$

$$
E=\frac{1}{l}\left(E_{\mathrm{b}}-R_{\mathrm{e}}\left(\frac{b H_{\mathrm{c}}}{2}-I_{\mathrm{l}}\right)\right)
$$

An inspection of Figure 8 shows that the flux distribution corresponding to such intermediate values of $E$ must result in an $N C, s C$, or $N s C$ region, according as $I_{1}$ is $>\frac{+a H_{\mathrm{c}}}{2}$, between $\frac{+a H_{\mathrm{c}}}{2}$ and $\frac{-a H_{\mathrm{c}}}{2}$ or $<\frac{-a H_{\mathrm{c}}}{2}$. Only by such a course can the $H r$ curve be made to end at point $N$, except of course when $I_{1}$ is so large and negative as to lead to the $N s$ type of region already treated. These $N C, s C$, and $\mathrm{NsC}$ regions, therefore, are stable and occupy a finite part of the threedimensional $E, I_{1}, I_{0}$ surface although they show only in projection in Figure 1. 
For the $N C$ region if $r_{\mathrm{o}}$ is the inner radius of the $C$ zons

$$
I_{0}=\frac{b H_{\mathrm{c}}}{2}-I_{1}=\pi E \lambda\left(r_{\mathrm{o}}^{2}-a^{2}\right)+\frac{H_{\mathrm{c}}}{2}\left(b-r_{\mathrm{o}}\right)
$$

whence

$$
E=\frac{1}{\pi \lambda\left(r_{\mathrm{o}}^{2}-a^{2}\right)}\left(\frac{H_{\mathrm{c}} r_{\mathrm{o}}}{2}-I_{1}\right)
$$

for $E=0, r_{\mathrm{o}}=\frac{2 I_{1}}{H_{\mathrm{c}}}$ and as $E$ increases to the value $\frac{H_{\mathrm{c}}}{4 \pi \lambda b}, r_{\mathrm{o}}$ increases to $b$.

For the $s C$ region the entire cross section is in the $C$ condition except for the current sheet at the inner surface.

For the $N s C$ region the radius $r_{\mathrm{o}}$ of the current sheet $s$ is related to the potential gradient by the equation

$$
\pi E \lambda\left(r_{\mathrm{o}}^{2}-a^{2}\right)=\frac{-H_{\mathrm{c}} r_{\mathrm{o}}}{2}-I_{1}
$$

for

for

$$
E=0, r_{0}=\frac{-2 I_{1}}{H_{\mathrm{c}}}
$$

$$
E=\frac{H_{\mathrm{c}}}{4 \pi \lambda b}, r_{\mathrm{o}}=b
$$

WAShington, March 22, 1927. 